\title{
Modeling reaction-diffusion of molecules on surface and in volume spaces with the E-Cell System
}

\author{
Satya Nanda Vel Arjunan*i and Masaru Tomita*†† \\ *Institute for Advanced Biosciences, Keio University, Tsuruoka, Yamagata, Japan \\ ${ }^{\dagger}$ Systems Biology Program, Graduate School of Media and Governance, Keio University, Fujisawa, Japan \\ ${ }^{\ddagger}$ Department of Environment and Information, Keio University, Fujisawa, Japan \\ satya@sfc.keio.ac.jp
}

\begin{abstract}
The E-Cell System is an advanced open-source simulation platform to model and analyze biochemical reaction networks. The present algorithm modules of the system assume that the reacting molecules are all homogeneously distributed in the reaction compartments, which is not the case in some cellular processes. The MinCDE system in Escherichia coli, for example, relies on intricately controlled reaction, diffusion and localization of Min proteins on the membrane and in the cytoplasm compartments to inhibit cell division at the poles of the rodshaped cell. To model such processes, we have extended the $\mathbf{E}$-Cell System to support reaction-diffusion and dynamic localization of molecules in volume and surface compartments. We evaluated our method by modeling the in vivo dynamics of MinD and MinE and comparing their simulated localization patterns to the observations in experiments and previous computational work. In both cases, our simulation results are in good agreement.
\end{abstract}

Keywords-lattice, hexagonal close-packed, systems biology, Monte Carlo, simulation, FtsZ, MinC

\section{INTRODUCTION}

$\mathbf{T}$ HE E-Cell System is one of the well known and advanced open-source simulation platforms to model and analyze both small- and large-scale biochemical reaction networks in living cells [1]. The driver algorithm of the E-Cell System (version 3) supports concurrent executions of multiple simulation algorithms, whose time steps are independently advanced in continuous-time, discrete-time or discrete-event manner at varying timescales [2]. Multiple sessions of simulations, usually required for estimation of reaction parameters and to obtain the averaged results from stochastic reactions, can be simultaneously executed with its distributed computing utility [3], [4]. Simulation runs can be automated and modified ex tempore with Python scripting, while new simulation algorithms can be developed using $\mathrm{C}++$ and incorporated into the system as plug in modules.

Recent advances in molecular biology suggest that modeling reaction networks alone is not sufficient to accurately reproduce certain important cellular processes such as cell division [5] and gene expression [6]. The dynamic location, crowding and diffusion of molecules in cellular compartments play

This work is supported by the Monbukagakusho Scholarship from the Ministry of Education, Culture, Sports, Science and Technology of Japan, Core Research for Evolutional Science and Technology (CREST) program by the Japan Science and Technology Agency (JST) and by the research funds from the Yamagata prefectural government and Tsuruoka City, Japan. crucial roles in such processes [7]. Molecular crowding can cause some subspace within a compartment to be inaccessible to reacting molecules because it is occupied by other macromolecules. This volume exclusion effect can reduce molecular diffusion and alter reaction kinetics [8].

In the rod-shaped bacterial cell Escherichia coli, the division site is restricted to the midcell by nucleoid occlusion and the pole-to-pole oscillation of the proteins $\mathrm{MinC}, \mathrm{MinD}$ and MinE, collectively called the MinCDE system (reviewed in [5]). The periodic oscillation is established because of intricately controlled reaction and diffusion of the proteins in the cytoplasm and the inner membrane. A simplified model of the system is illustrated in Figure I, which includes only MinD and MinE since MinC is not necessary for the oscillation. Division at the midpoint of the cell is important to ensure equal distribution of cell contents to the two daughter cells. The MinCDE molecules, found in low copies in the cytoplasm and on the inner cell membrane compartments, are not evenly distributed temporally. As a result, the rate of reactions, which is determined by the frequency of collision between reacting molecules, is influenced by diffusion and physical localization within the compartments. The molecules undergo surface (two-dimensional space) and volume (three-dimensional space) reaction-diffusion (RD) on the cell membrane and in the cytoplasm respectively.

Current algorithm modules of the E-Cell System assume that reactions take place between molecules that are uniformly distributed within the reaction compartment. It is also not possible to specify the physical location of each molecule. In this paper, we describe the extension of the E-Cell System to model the spatial localization and RD of molecules. Out of the several available methods (see Table I), ours is the only one that can account for the important implications of volume exclusion by molecules and RD in both volume and surface spaces. To evaluate the new approach, we compare the simulation results of the MinDE system with the results obtained from experiments and previous computational work.

\section{Methodology}

In this section, we describe the proposed RD scheme before providing the details of the implementation with the E-Cell System. 


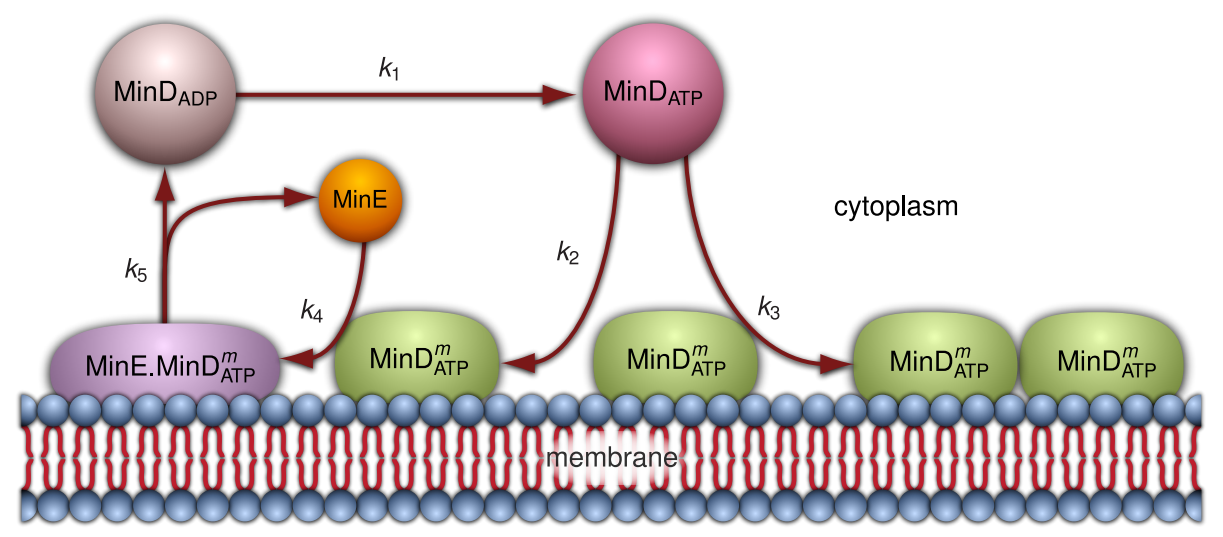

Figure 1. The simplified oscillation model of the MinDE sytem in Escherichia coli, adapted from [9]. MinC is not represented because it is not essential for the oscillation. Arrows depict the five basic reactions in the model. In the first reaction, MinD $\mathrm{ADP}_{\text {exchanges nucleotide to become MinD }}$ ATP with the rate $k_{1}$. In the form of $\mathrm{MinD}_{\mathrm{ATP}}$, the molecule can bind to the membrane either autonomously with the rate $k_{2}$ or cooperatively with a another membrane-bound $\mathrm{MinD}_{\mathrm{ATP}}^{m}$ at the rate $k_{3}$. Cytosolic MinE is also recruited to the membrane by $\mathrm{MinD}_{\mathrm{ATP}}^{m}$ with the rate $k_{4}$ to form MinE.MinD $\mathrm{ATP}^{m}$. The ATPase function of MinD is activated by MinE in the MinE.MinD $\mathrm{ATP}_{\mathrm{AT}}^{m}$ complex and consequently, MinD $\mathrm{ATP}_{\text {is }}$ converted to MinD $\mathrm{ADP}$ that cannot stay bound to the membrane. This is represented by the fifth reaction, in which the MinE.MinD $\mathrm{ATP}^{m}$ complex dissociates from the membrane at the rate $k_{5}$ and forms the cytosolic monomers MinE and MinD $\mathrm{ADP}$.

Table I

COMPARISON OF REACTION-DIFFUSION MODELING METHODS

\begin{tabular}{|l|c|c|c|}
\hline Name & $\begin{array}{c}\text { Volume } \\
\text { Exclusion }\end{array}$ & $\begin{array}{c}\text { Volume } \\
\text { RD }\end{array}$ & $\begin{array}{c}\text { Surface } \\
\text { RD }\end{array}$ \\
\hline MCell [10] & - & + & + \\
MesoRD [11], [12] & - & + & + \\
Smoldyn [13] & - & + & + \\
GMP [14] & - & + & + \\
GFRD [15] & + & + & - \\
CyberCell [16] & + & + & - \\
GridCell [17] & + & + & - \\
E-Cell (this work) & + & + & + \\
\hline
\end{tabular}

\section{A. The Reaction-Diffusion Scheme}

Molecules diffuse freely in space by making random walks [18]. According to the Collins and Kimball theory [19], when two reactive molecules come into contact (i.e., collide), they react with a certain probability $p$, which is related to the reaction rate constant $k$. To avoid molecule search when checking for collisions, we have discretized the space into hexagonal close-packed lattice [20]. Each sphere voxel in the lattice has 12 adjoining neighbor voxels. To account for volume exclusion and molecular crowding, each voxel can be occupied by a single molecule. The radius of the voxels is set according to the size of diffusing molecules. A molecule can walk to a randomly selected neighbor voxel in an interval $\tau_{d}$ following the Einstein-Smoluchowski's expression for diffusion

$$
\tau_{d}=\frac{\left\langle r^{2}\right\rangle}{2 l D},
$$

where $\left\langle r^{2}\right\rangle$ and $D$ are the mean squared displacement and the diffusion coefficient of the molecule respectively, and $l=2$ for surface diffusion while for volume diffusion, $l=3$. Since in the interval $\tau_{d}$ the molecule walks to a neighbor voxel, the mean squared displacement is given by the lattice spacing. We have derived the spacing for surface and volume diffusion, and the connection between $p$ and $k$ in [21]. At the destination voxel, the walking molecule may collide with another molecule that is a reactant pair and react if an independent random number drawn from a unit uniform distribution is less than $p$.

\section{B. E-Cell System Data Structure and Driver Algorithm}

The specific details of the E-Cell System data structure, driver and integration algorithms have previously been described [2]. We briefly provide the data structure and the driver algorithm here to characterize the implementation of our algorithm modules. We adopt the notations in [2] and capitalize the class names.

A reaction network system is represented in E-Cell as a Model, specified by the user in E-Cell Model description language (EML), a subset of XML. Figure 2 shows the data structure of the Model class, which contains a list of state Variables and Steppers. The Stepper class is the main algorithm module of the Model and it operates with a set of Processes, the current local time $\tau$ and the step interval $\Delta \tau$, a step method that advances the Stepper in time either in a continuous-time, discrete-time or discrete-event fashion, and an interruption method that allows other Steppers to notify the Stepper when they modify a read Variable of the Stepper. The Process is a lower level algorithm module that directly reads or modifies the state Variables according to the algorithm using a transition-function. Here, the instances of the state Variables are accessed by dereferencing the Variable References.

Central to the E-Cell driver algorithm is a priority queue that arranges the Steppers according to the scheduled time of execution, given as $\tau+\Delta \tau$. At initialization, the global time $t$ and the local time $\tau$ of Steppers are reset. Next, the step method of the Stepper $S_{i}$ with the minimum scheduled time is called and the global time is updated to $t=\tau_{i}+\Delta \tau_{i}$. The step method also sets the local time to $\tau_{i}=\tau_{i}+\Delta \tau_{i}$ and calls the transition function of its Processes to update the state Variables. The method may also update the next step size $\Delta \tau_{i}$ 
and call the interruption method of other Steppers whose read Variable has been modified. The Stepper $S_{i}$ is rescheduled in the priority queue according to the new scheduled time. The same procedure is repeated for the Stepper with the next earliest scheduled time in the priority queue until the simulation is ended.

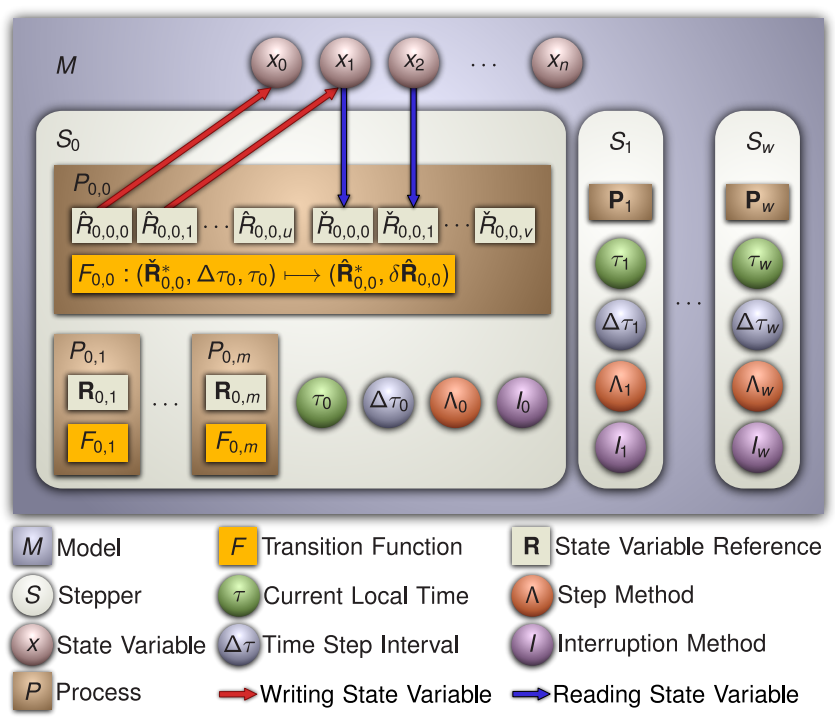

Figure 2. The data structure of the E-Cell Model.

\section{Implementation of Reaction-Diffusion with E-Cell}

We have implemented the proposed reaction-diffusion scheme using the E-Cell System by creating two basic algorithm modules - a Diffusion Process and a Reaction Process. The molecules are represented as the state Variables of the Model. For each diffusion coefficient in simulation, a Diffusion Process object is created to walk the molecules. Likewise, a Reaction Process object is instantiated for every reaction involving a diffusing molecule. A discrete-event Stepper advances the Diffusion Process in time and handles the lattice structure and the physical location of molecules. The transition function of the Diffusion Process walks each molecule to a randomly selected adjoining voxel in a diffusion step interval. When a molecule collides with a reactant pair, the transition function of the corresponding Reaction Process is called. If the reactive collision probability is met, the Process removes the collided molecules and replaces them with one or two product molecules, as specified by the reaction.

\section{ApPliCATION Results}

We modeled the periodic oscillatory behavior of molecules in the MinDE system to evaluate our approach because it involves both surface and volume RD, and spatio-temporal localization of molecules. We describe the computational model of the sytem before presenting the results of simulation.

\section{A. The MinDE Model}

In Escherichia coli, the FtsZ membrane protein initiates cell division physically by polymerizing and constricting annularly at the middle of the long axis of the rod-shaped cell [5], [22]. Although the protein can diffuse over the entire membrane, nucleoid occlusion prevents the polymerization from taking place over the nucleoid mass, leaving only the midcell and the two cell poles as viable locations for polymerization [23], [24], [25]. Nonetheless, because of the inhibition by MinC proteins at the poles [26], [27], the polymerization can only take place at the midcell. During the cell cycle, MinD with the help of MinE forms polar zones which oscillate from one pole to the other. Since MinC piggybacks on MinD and the oscillation occurs on the rod-shaped cell with some dwelling time at the poles, the time-averaged concentration of MinC at the middle of the cell is kept low, permitting FtsZ to polymerize.

According to the model by Huang et al. [9] (illustrated in Figure I) cytosolic MinD in the ATP-bound form $\left(\mathrm{MinD}_{\mathrm{ATP}}\right)$ binds to the membrane either cooperatively with another membrane bound MinD $\left(\mathrm{MinD}_{\mathrm{ATP}}^{m}\right)$ or independently. MinE from the cytoplasm inhibits $\mathrm{MinD}_{\mathrm{ATP}}^{m}$ by first associating with it and setting off the ATPase function of MinD that hydrolyzes the bound ATP to ADP. The membrane-bound MinE and the ADP-bound form of MinD ( $\left.\operatorname{MinD}_{\mathrm{ADP}}\right)$ then dissociate to the cytoplasm. In the cytoplasm, $\mathrm{MinD}_{\mathrm{ADP}}$ is phosphorylated and takes the form of $\mathrm{MinD}_{\mathrm{ATP}}$ again. MinC is not explicitly represented in the model because it is usually attached to MinD and experimental data indicate that the oscillation can take place without it [27], [28]. The series of reactions in the model are as follows:

$$
\begin{gathered}
\operatorname{MinD}_{\mathrm{ADP}} \stackrel{k_{1}}{\longrightarrow} \operatorname{MinD}_{\mathrm{ATP}} \\
\operatorname{MinD}_{\mathrm{ATP}} \stackrel{k_{2}}{\longrightarrow} \operatorname{MinD}_{\mathrm{ATP}}^{m} \\
\operatorname{MinD}_{\mathrm{ATP}}+\operatorname{MinD}_{\mathrm{ATP}}^{m} \stackrel{k_{3}}{\longrightarrow} 2 \operatorname{MinD}_{\mathrm{ATP}}^{m} \\
\operatorname{MinE}+\operatorname{MinD}_{\mathrm{ATP}}^{m} \stackrel{k_{4}}{\longrightarrow} \operatorname{MinE} \operatorname{MinD}_{\mathrm{ATP}}^{m} \\
\operatorname{MinE} \cdot \mathrm{MinD}_{\mathrm{ATP}}^{m} \stackrel{k_{5}}{\longrightarrow} \operatorname{MinE}+\operatorname{MinD}_{\mathrm{ADP}}
\end{gathered}
$$

\section{B. Simulation Results and Discussion}

To simulate the MinDE oscillation model using the proposed approach, we applied the parameters from [29] (listed in Table II). However, we reduced the radius of our voxels to more closely reflect the size of diffusing molecules. Another variation between our method and the MesoRD method in [29] is that our molecules can exhibit excluded volume.

Figure 3 shows the random distribution of cytosolic $\operatorname{MinD}_{\mathrm{ADP}}, \mathrm{MinD}_{\mathrm{ATP}}$ and MinE molecules at initialization. The pole-to-pole oscillation of membrane-bound $\mathrm{MinD}_{\mathrm{ATP}}^{m}$, as shown in Figure 4, is spontaneously triggered after about 1 minute of simulated time although all molecules were initially randomly distributed in the cytoplasm. For the 1 minute of simulated time, it takes about 14 minutes of simulation on an Intel Core 2 Extreme $3.2 \mathrm{GHz}$ system with $8 \mathrm{~GB}$ of RAM. The oscillation has an average period of 36 seconds which corresponds to what has been observed experimentally 
[30], [28]. The period is also in close agreement with the value from the previously reported computational model [29]. Consistent with the observations by Huang et al. [9] and in [29], the period increased proportionally to the number of MinD, while reduced proportionally to the amount of MinE in the model. In addition, as observed in the MinD-MinE localization studies in Escherichia coli [31], the membranebound MinE.MinD $\mathrm{ATP}_{\mathrm{AT}}^{m}$ dimers appear to be lagging behind $\mathrm{MinD}_{\mathrm{ATP}}^{m}$ molecules when they migrate from one pole to the other. Taken together, our simulations closely reproduce the results from both experimental and previous computational studies.

Table II

PARAMETERS OF THE MINDE MODEL

\begin{tabular}{|l|l|l|}
\hline Variable & Value & Units \\
\hline$k_{1}$ & 0.5 & $\mathrm{~s}^{-1}$ \\
$k_{2}$ & 0.0125 & $\mu \mathrm{ms}^{-1}$ \\
$k_{3}$ & 0.0149 & $\mu \mathrm{m}^{3} \mathrm{~s}^{-1}$ \\
$k_{4}$ & 0.0923 & $\mu \mathrm{m}^{3} \mathrm{~s}^{-1}$ \\
$k_{5}$ & 0.7 & $\mathrm{~s}^{-1}$ \\
$D_{\text {cytoplasm }}$ & 2.5 & $\mu \mathrm{m}^{2} \mathrm{~s}^{-1}$ \\
$D_{\text {membrane }}$ & 0.01 & $\mu \mathrm{m}^{2} \mathrm{~s}^{-1}$ \\
Cell volume & 3.27 & $\mu \mathrm{m}^{3}$ \\
Cell radius & 0.5 & $\mu \mathrm{m}$ \\
Voxel radius & 8 & $\mathrm{~nm}$ \\
Initial MinD ATP molecules & 2001 & \\
Initial MinD ADP molecules & 2001 & \\
Initial MinE molecules & 1040 & \\
\hline
\end{tabular}

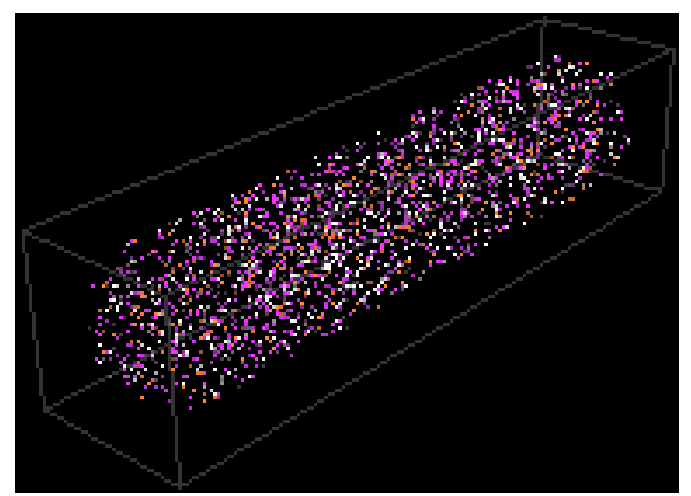

Figure 3. Simulated cytosolic molecules of the MinDE system. MinD $D_{\text {ATP }}$ (purple), MinD $\mathrm{ADP}$ (white) and MinE (red) are randomly distributed in the cytoplasm of Escherichia coli at initialization.

Here, we describe the steps that trigger the spontaneous oscillation. Initially, all molecules are evenly distributed in the cytoplasm. Very small number of MinD $_{\text {ATP }}$ molecules begin to independently associate at random locations on the membrane and rigorously recruit other MinD $_{\mathrm{ATP}}$ molecules cooperatively. As shown in Figure 5, the recruitment gives the appearance of growing patches on the membrane. Cytosolic MinE molecules are attracted to these patches because of their high affinity to $\mathrm{MinD}_{\mathrm{ATP}}^{m}$ and form MinE.MinD $\mathrm{ATP}_{\mathrm{AT}}^{m}$ dimers. Soon the patches loosely cover the entire membrane because the rate of MinD recruitment is faster than the rate of dissociation, even after almost all MinE are found in the
MinE.MinD $D_{\text {ATP }}^{m}$ dimer form on the membrane. At random locations on the membrane, some $\mathrm{MinD}_{\mathrm{ATP}}^{m}$ patches are free from MinE inhibition because of the limited cytosolic MinE molecules. In addition, these patches also become more persistent at locations farther from the dissociating patches, where they are less inhibited by the newly released MinE molecules and where $\mathrm{MinD}_{\mathrm{ATP}}$ can escape cooperative recruitment by $\mathrm{MinD}_{\mathrm{ATP}}^{m}$ in the dissociating patches. Finally, patches (or polar zones) form alternately (i.e., oscillate) at the two poles of the cell because the poles are sufficiently far from each other to avoid both the rapid inhibition by the released MinE and the cooperative recruitment by $\mathrm{MinD}_{\mathrm{ATP}}^{m}$. During the oscillation cycle, the MinE.MinD ${ }_{\text {ATP }}^{m}$ dimers appear to be lagging behind $\mathrm{MinD}_{\mathrm{ATP}}^{m}$ because the released MinE molecules from the opposite pole find $\mathrm{MinD}_{\mathrm{ATP}}^{m}$ at the rim of the polar zone first.

From the simulations, we observed several important features of the MinDE system that support the periodic oscillations. When we increased the nucleotide exhange rate of $\mathrm{MinD}_{\mathrm{ADP}}$ to $k_{1}=1 \mathrm{~s}^{-1}$, the population of $\mathrm{MinD}_{\mathrm{ATP}}$ increased, resulting in more $\mathrm{MinD}_{\mathrm{ATP}}^{m}$ and longer polar zones. Since there are more $\mathrm{MinD}_{\mathrm{ATP}}^{m}$ to be activated by MinE, the oscillation period also increased to about $63 \mathrm{~s}$. Conversely, the duration of an oscillation cycle is reduced to approximately 20 $\mathrm{s}$ when the rate is decreased to $k_{1}=0.3 \mathrm{~s}^{-1}$ because of the limited number of $\mathrm{MinD}_{\mathrm{ATP}}$ copies available.

The reactive collision probability $p$ for $\mathrm{MinD}_{\mathrm{ATP}}$ to associate independently to the lipid molecules on the membrane $\left(k_{2}=0.0125 \mu \mathrm{ms}^{-1}, p=0.274 \times 10^{-4}\right)$ is about four orders of magnitude lower than to associate cooperatively with another membrane-bound $\operatorname{MinD}_{\mathrm{ATP}}^{m}\left(k_{3}=\right.$ $\left.0.0149 \mu \mathrm{m}^{3} \mathrm{~s}^{-1}, \quad p=0.16\right)$, even though the number of lipid molecules $(\sim 72000)$ is only about an order of magnitude more than that of $\operatorname{MinD}_{\mathrm{ATP}}^{m}(\sim 1400)$. This ensures that $\mathrm{MinD}_{\mathrm{ATP}}$ only binds to the membrane independently to nucleate bindings of other MinD $\mathrm{ATP}_{\mathrm{AT}}$. When we increased $k_{2}=0.05 \mu \mathrm{ms}^{-1}, \mathrm{MinD}_{\mathrm{ATP}}^{m}$ were found loosely distributed in the polar zone that extended beyond the midcell because the enhanced nucleation rate allows $\mathrm{MinD}_{\mathrm{ATP}}$ to successfuly bind almost anywhere on the membrane. Occasionally, there were no clear definition of the polar zones, with $\mathrm{MinD}_{\mathrm{ATP}}^{m}$ and MinE.MinD $\mathrm{ATP}_{\mathrm{AT}}^{m}$ covering the entire membrane. The oscillation period increased moderately to about $44 \mathrm{~s}$. Reducing $k_{2}=0.0009 \mu \mathrm{ms}^{-1}$ shortened the period marginally to 33 $\mathrm{s}$ and displayed erratic nucleation patterns, with the polar zones frequently appearing and growing near the midcell. In addition, because of the decreased nucleation rate, cooperative membrane recruitments of $\mathrm{MinD}_{\mathrm{ATP}}$ dominated further and rigorous membrane associations occured at the nucleation sites.

When the rate of cooperative recruitment was increased to $k_{3}=0.034 \mu \mathrm{m}^{3} \mathrm{~s}^{-1}$, the oscillation was not triggered because MinE cannot rapidly activate the rigorously recruited $\mathrm{MinD}_{\mathrm{ATP}}^{m}$. Therefore, both $\mathrm{MinD}_{\mathrm{ATP}}^{m}$ and MinE.MinD $\mathrm{ATP}^{m}$ were uniformly distributed on the membrane. Setting $k_{3}=$ $0.024 \mu \mathrm{m}^{3} \mathrm{~s}^{-1}$ stimulated the oscillation but the polar zones were not clearly defined and extended well over the midcell because of the larger population of $\mathrm{MinD}_{\mathrm{ATP}}^{m}$. The oscillation period also increased to approximately $50 \mathrm{~s}$. Reduc- 
ing $k_{3}=0.004 \mu \mathrm{m}^{3} \mathrm{~s}^{-1}$ also prevented the oscillation because $\mathrm{MinD}_{\mathrm{ATP}}$ cannot successfully bind to the membraneMinE rapidly dissociates them since their cooperative recruitment activity has been weakened. However, setting $k_{3}=$ $0.007 \mu \mathrm{m}^{3} \mathrm{~s}^{-1}$ started the oscillation with a reduced period of about $30 \mathrm{~s}$, while the polar zones were occasionally nucleated near the midcell.

Reducing or increasing the MinE membrane recruitment rate $k_{4}$ has generally the opposite effect of $k_{3}$. This is because the membrane associated MinE activates MinD ATPase function that dissociates MinD to the cytoplasm. Increasing the rate to $k_{4}=0.4 \mu \mathrm{m}^{3} \mathrm{~s}^{-1}$ generated the oscillation with a shortened period of about $30 \mathrm{~s}$ since more MinE.MinD $\mathrm{ATP}_{\mathrm{AT}}^{m}$ are available to activate MinD. The polar zones were nucleated both near the midcell and at the poles, resulting in their rapid growth in each cycle. Conversely, setting $k_{4}=0.05 \mu \mathrm{m}^{3} \mathrm{~s}^{-1}$ produced polar zones that are not clearly defined with an oscillation period of approximately $41 \mathrm{~s}$. Both polar zones frequently appeared simultaneously with one usually covering more than one half of the cell long axis.

The MinE.MinD $D_{\text {ATP }}^{m}$ dissociation rate $k_{5}$ has almost the same properties as $k_{4}$ but with higher efficacy. When the rate was increased to $k_{5}=1.2 \mathrm{~s}^{-1}$, the polar zones were shorter and cycled between the poles with a period lasting approximately $12 \mathrm{~s}$. The higher rate increases the population of free MinE that can associate and activate other $\mathrm{MinD}_{\mathrm{ATP}}^{m}$, thus reducing the size of the polar zones and increasing the oscillation speed. On the other hand when the rate was reduced to $k_{5}=0.5 \mathrm{~s}^{-1}$, the period increased to about $54 \mathrm{~s}$, while the polar zones were nucleated at the cell poles and extended beyond the midcell.

The significantly slower diffusion coefficient of $\mathrm{MinD}_{\mathrm{ATP}}^{m}$ and MinE.MinD $\mathrm{ATP}_{\mathrm{AT}}^{m}$ on the membrane prohibits the molecules from rapidly achieving uniform concentration on the membrane. By increasing the diffusion coefficient twofold to $D_{\text {membrane }}=0.02 \mu \mathrm{m}^{2} \mathrm{~s}^{-1}$, the polar zones extended beyond the midcell and were not clearly defined. The oscillation period was about $60 \mathrm{~s}$. Increasing the coefficient further to $D_{\text {membrane }}=0.05 \mu \mathrm{m}^{2} \mathrm{~s}^{-1}$ did not produce the oscillation.

\section{CONCLusions}

We have successfully extended the E-Cell System to model $\mathrm{RD}$ on surface and in volume spaces with dynamic localization of molecules. A unique feature of our method is that it can account for the important implications of volume exclusion by molecules while performing RD in both volume and surface spaces [21]. The correctness of our method and implementation is demonstrated by the accurate reproduction of the MinD and MinE oscillation behaviors in Escherichia coli, as observed in both experimental and previous computational studies. We modeled the MinDE system because the proteins display unique properties such as spatio-temporal localization patterns on the membrane and inter-compartmental reactions. We have shown the impact of changing the various reaction and diffusion parameters to the dynamic localization patterns of the proteins. Recent experimental studies have shown that MinD forms helical polymers on the membrane [32]. Our

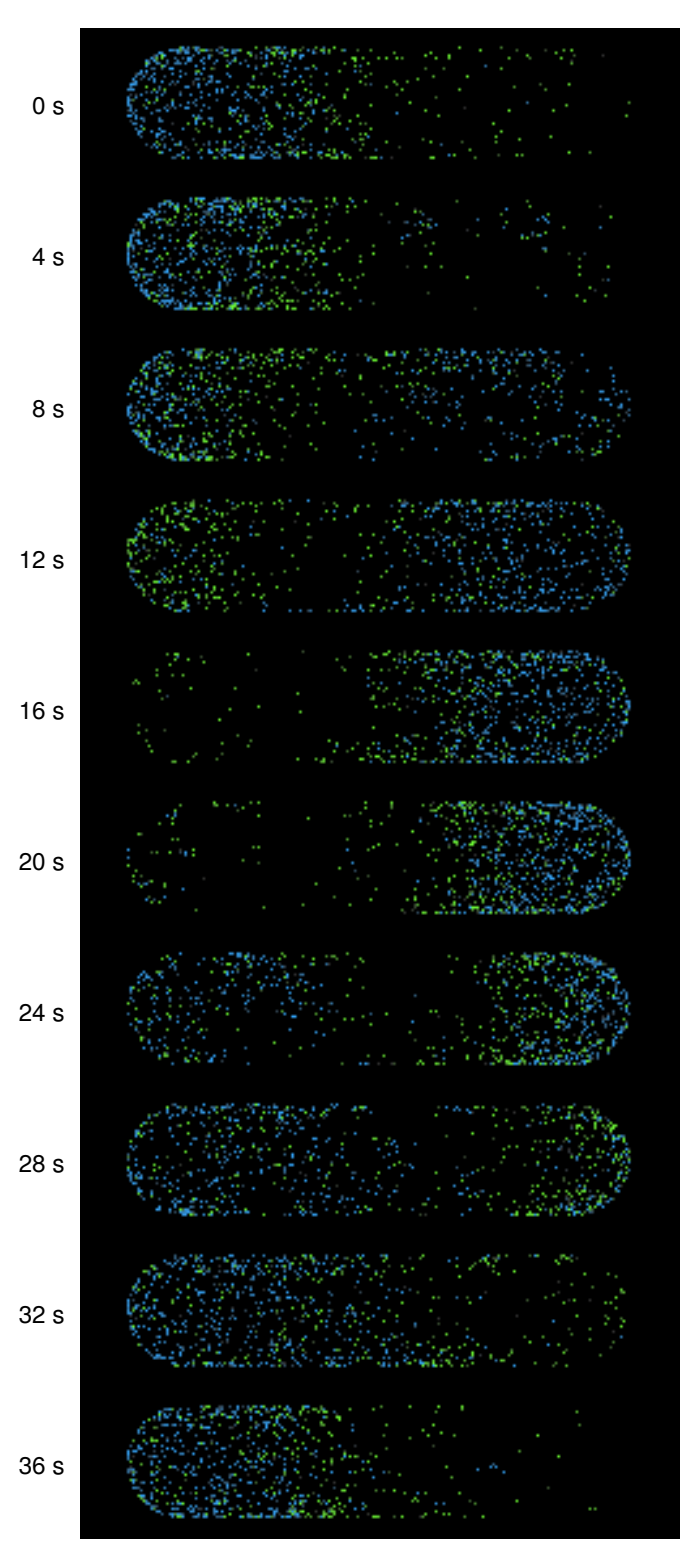

Figure 4. MinD and MinE oscillation on the membrane of Escherichia coli. MinD $\mathrm{ATP}_{\mathrm{ATP}}^{m}$ monomers and MinE.MinD $\mathrm{ATP}_{\mathrm{AT}}^{m}$ dimers are shown in cyan and green respectively. The $\mathrm{MinD}_{\mathrm{ATP}}^{m}$ monomers appear to lead the dimers in the oscillation cycle which has an average period of 36 seconds.

work can be further extended to model such membranebound polymerization dynamics of molecules. The software implementation of the method and the model presented in this paper are provided upon request. A detailed guide for the $\mathrm{RD}$ modeling using the method is also available [33].

\section{ACKNOWLEDGMENT}

We thank Martin Robert, Koichi Takahashi, Takeshi Sakurada, Mohamed Helmy and Moriyoshi Koizumi for useful discussions.

\section{REFERENCES}

[1] M. Tomita, K. Hashimoto, K. Takahashi, T. S. Shimizu, Y. Matsuzaki, F. Miyoshi, K. Saito, S. Tanida, K. Yugi, J. C. Venter, and C. A. Hutchison, "E-CELL: software environment for whole-cell simulation," Bioinformatics, vol. 15, no. 1, pp. 72-84, 1999. 


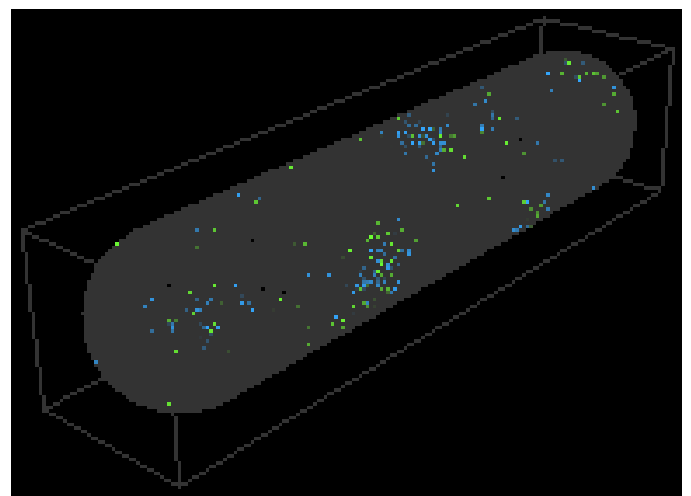

Figure 5. Initial patches of MinD (cyan) and MinE.MinD ATP (green) forming on the membrane (gray).

[2] K. Takahashi, K. Kaizu, B. Hu, and M. Tomita, "A multi-algorithm, multi-timescale method for cell simulation," Bioinformatics, vol. 20, no. 4, pp. 538-546, Mar. 2004

[3] M. Sugimoto, K. Takahashi, T. Kitayama, D. Ito, and M. Tomita, "Distributed cell biology simulations with E-Cell system," in Grid Computing in Life Science, ser. Lecture Notes in Computer Science, 2005, vol. 3370/2005, pp. 20-31.

[4] M. Sugimoto, "Distributed cell biology simulations with the E-Cell system," in E-Cell System: Basic Concepts and Applications, ser. Intelligence Unit, S. N. V. Arjunan, P. K. Dhar, and M. Tomita, Eds. Georgetown, Texas: Landes Bioscience and Springer Science+Business Media, Aug. 2009.

[5] J. Lutkenhaus, "Assembly dynamics of the bacterial MinCDE system and spatial regulation of the $\mathrm{Z}$ ring," Annu. Rev. Biochem, vol. 76, pp. 539-562, 2007.

[6] P. B. Talbert and S. Henikoff, "Spreading of silent chromatin: inaction at a distance," Nat. Rev. Genet, vol. 7, no. 10, pp. 793-803, Oct. 2006

[7] K. Takahashi, S. N. V. Arjunan, and M. Tomita, "Space in systems biology of signaling pathways-towards intracellular molecular crowding in silico," FEBS Lett, vol. 579, no. 8, pp. 1783-1788, Mar. 2005.

[8] R. J. Ellis, "Macromolecular crowding: obvious but underappreciated," Trends Biochem. Sci, vol. 26, no. 10, pp. 597-604, Oct. 2001

[9] K. C. Huang, Y. Meir, and N. S. Wingreen, "Dynamic structures in Escherichia coli: spontaneous formation of MinE rings and MinD polar zones," Proc. Natl. Acad. Sci. USA, vol. 100, no. 22, pp. 12 724-12728, Oct. 2003.

[10] J. R. Stiles and T. M. Bartol, "Monte Carlo methods for simulating realistic synaptic microphysiology using MCell," in Computational Neuroscience: Realistic Modeling for Experimentalists, E. D. Schutter, Ed. Boca Raton, FL: CRC Press, Nov. 2001, pp. 87-127.

[11] J. Elf and M. Ehrenberg, "Spontaneous separation of bi-stable biochemical systems into spatial domains of opposite phases," Syst. Biol, vol. 1, no. 2, pp. 230-236, Dec. 2004

[12] J. Hattne, D. Fange, and J. Elf, "Stochastic reaction-diffusion simulation with MesoRD," Bioinformatics, vol. 21, no. 12, pp. 2923-2924, Jun. 2005.

[13] S. S. Andrews and D. Bray, "Stochastic simulation of chemical reactions with spatial resolution and single molecule detail," Phys. Biol, vol. 1, no. 3-4, pp. 137-151, Dec. 2004.

[14] J. V. Rodríguez, J. A. Kaandorp, M. Dobrzyński, and J. G. Blom, "Spatial stochastic modelling of the phosphoenolpyruvate-dependent phosphotransferase (PTS) pathway in Escherichia coli," Bioinformatics, vol. 22, no. 15, pp. 1895-1901, Aug. 2006.

[15] J. S. van Zon and P. R. ten Wolde, "Simulating biochemical networks at the particle level and in time and space: Green's function reaction dynamics," Phys. Rev. Lett, vol. 94, no. 12, p. 128103, Apr. 2005.

[16] D. Ridgway, G. Broderick, A. Lopez-Campistrous, M. Ru'aini, P. Winter, M. Hamilton, P. Boulanger, A. Kovalenko, and M. J. Ellison, "Coarsegrained molecular simulation of diffusion and reaction kinetics in a crowded virtual cytoplasm," Biophys. J, vol. 94, no. 10, pp. 3748-3759, May 2008.

[17] L. Boulianne, S. A. Assaad, M. Dumontier, and W. Gross, "GridCell: a stochastic particle-based biological system simulator," BMC Syst. Biol, vol. 2 , no. 1, p. 66, Jul. 2008.
[18] H. C. Berg, Random walks in biology. Princeton University Press, 1993.

[19] F. C. Collins and G. E. Kimball, "Diffusion-controlled reaction rates," J. Colloid Sci, vol. 4, no. 4, pp. 425-437, Aug. 1949.

[20] J. H. Conway and N. J. A. Sloane, Sphere Packings, Lattices and Groups, 3rd ed. Springer, Dec. 1998.

[21] S. N. V. Arjunan and M. Tomita, "A lattice-based method for volume and surface reaction-diffusion reproduces the MinE ring by cooperative activation of MinD," Submitted.

[22] J. Löwe and L. A. Amos, "Evolution of cytomotive filaments: the cytoskeleton from prokaryotes to eukaryotes," Int. J. Biochem. Cell Biol, vol. 41, no. 2, pp. 323-329, Feb. 2009

[23] C. L. Woldringh, E. Mulder, J. A. Valkenburg, F. B. Wientjes, A. Zaritsky, and N. Nanninga, "Role of the nucleoid in the toporegulation of division," Res. Microbiol, vol. 141, no. 1, pp. 39-49, 1990.

[24] C. L. Woldringh, E. Mulder, P. G. Huls, and N. Vischer, "Toporegulation of bacterial division according to the nucleoid occlusion model," Res. Microbiol, vol. 142, no. 2-3, pp. 309-320, Apr. 1991.

[25] T. G. Bernhardt and P. A. J. de Boer, "SlmA, a nucleoid-associated, FtsZ binding protein required for blocking septal ring assembly over chromosomes in E. coli," Mol. Cell, vol. 18, no. 5, pp. 555-564, May 2005.

[26] Z. Hu, A. Mukherjee, S. Pichoff, and J. Lutkenhaus, "The MinC component of the division site selection system in Escherichia coli interacts with FtsZ to prevent polymerization," Proc. Natl. Acad. Sci. U.S.A, vol. 96, no. 26, pp. 14819-14 824, Dec. 1999.

[27] Z. Hu and J. Lutkenhaus, "Topological regulation of cell division in Escherichia coli involves rapid pole to pole oscillation of the division inhibitor MinC under the control of MinD and MinE," Mol. Microbiol, vol. 34, no. 1, pp. 82-90, Oct. 1999.

[28] D. M. Raskin and P. A. de Boer, "Rapid pole-to-pole oscillation of protein required for directing division to the middle of Escherichia coli," Proc. Natl. Acad. Sci. USA, vol. 96, no. 9, pp. 4971-4976, Apr. 1999.

[29] D. Fange and J. Elf, "Noise-induced Min phenotypes in E. coli," PLoS Comput. Biol, vol. 2, no. 6, p. e80, Jun. 2006.

[30] C. A. Hale, H. Meinhardt, and P. A. de Boer, "Dynamic localization cycle of the cell division regulator MinE in Escherichia coli," EMBO J, vol. 20, no. 7, pp. 1563-1572, Apr. 2001.

[31] Y. Shih, X. Fu, G. F. King, T. Le, and L. Rothfield, "Division site placement in E.coli: mutations that prevent formation of the MinE ring lead to loss of the normal midcell arrest of growth of polar MinD membrane domains," EMBO J, vol. 21, no. 13, pp. 3347-3357, Jul. 2002.

[32] Y. Shih, T. Le, and L. Rothfield, "Division site selection in Escherichia coli involves dynamic redistribution of Min proteins within coiled structures that extend between the two cell poles," Proc. Natl. Acad. Sci. USA, vol. 100, no. 13, pp. 7865-7870, Jun. 2003.

[33] S. N. V. Arjunan, "A guide to modeling reaction-diffusion of molecules with the E-Cell System," in E-Cell System: Basic Concepts and Applications, ser. Intelligence Unit, S. N. V. Arjunan, P. K. Dhar, and M. Tomita, Eds. Georgetown, Texas: Landes Bioscience and Springer Science+Business Media, Aug. 2009 .

Satya Nanda Vel Arjunan is a Ph.D candidate in the Systems Biology Program at the Graduate School of Media and Governance in Keio University. He received his B.Eng. (2000) in Electronics Engineering and M.Sc. (2003) in Computer Science from Universiti Teknologi Malaysia. He is the recipient of the Texas Instruments Malaysia Scholarship (1997) and the Monbukagakusho Scholarship (2003)

Masaru Tomita is a Professor and the Director of the Institute for Advanced Biosciences, Keio University. He received his B.S (1981) in Mathematics from Keio University, M.S (1983) and Ph.D (1985) in Computer Science from Carnegie Mellon University, and another Ph.D (1994) in Molecular Biology from Keio University. Among other awards and prizes, Dr. Tomita is a recipient of the Presidential Young Investigators Award from National Science Foundation of USA (1988) and IBM Japan Science Prize (2002). 